\title{
NMR Study of 1,4-Dihydropyridine Derivatives Endowed with Long Alkyl and Functionalized Chains
}

\author{
Margarita Suárez, ${ }^{*}, a$ Dolores Molero, ${ }^{b}$ Esperanza Salfrán, ${ }^{a}$ Hortensia Rodríguez, ${ }^{a}$ \\ Julieta Coro, ${ }^{a}$ Elena Sáez, ${ }^{b}$ Roberto Martínez-Álvarez ${ }^{c}$ and Nazario Martín ${ }^{c}$ \\ ${ }^{a}$ Laboratorio de Síntesis Orgánica, Facultad de Química, Universidad de La Habana, 10400 \\ Ciudad Habana, Cuba \\ ${ }^{b}$ CAI-RMN and ${ }^{c}$ Departamento de Química Orgánica I, Facultad de Química, \\ Universidad Complutense, E-28040 Madrid, Spain
}

\begin{abstract}
Neste trabalho, realizou-se um estudo por ressonância magnética nuclear de ${ }^{1} \mathrm{H},{ }^{13} \mathrm{C}$ e ${ }^{15} \mathrm{~N}$ de derivados de 1,4-dihidropiridinas que contêm, nos grupos ésteres das posições C-3 e C-5, longas cadeias alquílicas ou funcionalizadas. Atribuíram-se inequivocamente os sinais dos espectros utilizando experimentos 1D e 2D (DEPT, nOe, HMQC, HMBC, COSY).
\end{abstract}

The ${ }^{1} \mathrm{H},{ }^{13} \mathrm{C}$ and ${ }^{15} \mathrm{~N}$ NMR spectroscopic data for 1,4-dihydropyridine endowed with long alkyl and functionalized chain on C-3 and C-5, have been fully assigned by combination of one- and two dimensional experiments (DEPT, HMBC, HMQC, COSY, nOe).

Keywords: ${ }^{1} \mathrm{H}$ NMR, ${ }^{13} \mathrm{C}$ NMR, ${ }^{15} \mathrm{~N}$ NMR, 1,4-dihydropyridines

\section{Introduction}

Throughout the recent years the synthesis and structural characterization of novel analogues of 1,4-dihydropyridines (1,4-DHPs) derivatives has received particular attention due to the interesting pharmacological and biological properties that they display., ${ }^{1,2}$ It is well known that the 1,4-DHP nucleus serves as scaffold for important cardiovascular drugs, and that the calcium modulating activity is determined by structural requirements. ${ }^{3}$ It has been proved that 1,4-DHP-calcium antagonists with a high degree of lipophilicity display a slow onset action after administration as vasodilator and hypertensive medications. ${ }^{4,5}$

In order to gain a better understanding of the effect of the substitution pattern of the 1,4-DHPs on biological activity, we have prepared several new 1,4-DHPs endowed with long alkyl and functionalised chains. ${ }^{6}$ In addition we have reported the study of the ESI mass spectra and sequential product ion fragmentation of 1,4-DHP derivatives endowed with long alkyl chains. ${ }^{7}$

In connection with our studies of the NMR behaviour of 1,4-dihydropyridine derivatives, in this paper reports

\footnotetext{
*e-mail: msuarez@fq.uh.cu
}

the ${ }^{1} \mathrm{H}$ and ${ }^{13} \mathrm{C}$ assignments of a series of differently substituted 1,4-dihydropyridines endowed with long and functionalized chains, 1a-d, 2a-b, 3a-e, 4a-c and 5a-b (see Figure 1). Also we describe the ${ }^{15} \mathrm{~N}$ NMR spectra of some chosen compounds. These data are of interest to determine the effect that this substitution pattern has on the chemical shifts of the 1,4-DHPs ring and to complete the reference data on the previously reported related systems. ${ }^{8,11}$

\section{Results and Discussion}

The 1,4-DHPs derivatives here studied were prepared by a Hantzsch-like synthesis, and obtained as stable crystalline solids with good yields.

In order to assign unequivocally all NMR signals, we used 1D and 2D techniques such as DEPT(135), HMQC and $\mathrm{HMBC}$.

The ${ }^{1} \mathrm{H}$ NMR spectra at $300 \mathrm{MHz}$ of 1,4-DHPs (Table 1) show a singlet corresponding to the $\mathrm{NH}$ proton at 9.19-8.76 ppm when the spectra were registered in DMSO- $d_{6}$ or at 6.55-7.97 when they were registered in $\mathrm{CDCl}_{3}$. Also at 4.75-5.02 ppm appears another singlet corresponding to the $\mathrm{H} 4$ proton.

The methyl groups on C-2 and C- 6 of the 1,4-DHP ring appear as singlets at $\delta c a .2 .2 \mathrm{ppm}$, and cannot be 


$$
\begin{aligned}
& \text { (1) } \\
& \text { 1a } \mathrm{R}=\stackrel{15}{\mathrm{C}} \mathrm{H}_{3} \\
& \text { 1b } \mathrm{R}=\stackrel{15}{\mathrm{C}} \mathrm{H}_{2}-\mathrm{CH}_{3} \\
& \text { 1c } \mathrm{R}=\stackrel{15}{\mathrm{C}} \mathrm{H}_{2}-\mathrm{CH}_{2}-\mathrm{CN} \\
& \text { 1d } \mathrm{R}=\stackrel{7}{\mathrm{C}} \mathrm{H}_{2}-\stackrel{8}{\mathrm{C}} \mathrm{H}_{2}-\stackrel{9}{\mathrm{C}} \mathrm{H}_{2}-\stackrel{10}{\mathrm{C}} \mathrm{H}_{2}-\stackrel{11}{\mathrm{C}} \mathrm{H}_{2}-\stackrel{12}{\mathrm{C}} \mathrm{H}_{2}-\stackrel{13}{\mathrm{C}} \mathrm{H}_{2}-\stackrel{14}{\mathrm{C}} \mathrm{H}_{3}
\end{aligned}
$$<smiles>[Z]CCC=CCCCCOC(=O)C1=C(C)NC(C)=C(C(=O)O[R])C1c1cccnc1</smiles>

$$
\begin{aligned}
& \text { 3a } \quad \mathrm{R}=\stackrel{15}{\mathrm{C}} \mathrm{H}_{3} \\
& \text { 3b } \quad \mathrm{R}=\stackrel{15}{\mathrm{C}} \mathrm{H}_{2}-\mathrm{CH}_{3} \\
& \text { 3c } \mathrm{R}=\stackrel{15}{\mathrm{C}} \mathrm{H}_{2}-\stackrel{16}{\mathrm{C}} \mathrm{H}_{2}-\stackrel{17}{\mathrm{C} N} \\
& \text { 3d } \mathrm{R}=\stackrel{7}{\mathrm{C}} \mathrm{H}_{2}-\stackrel{8}{\mathrm{C}} \mathrm{H}_{2}-\stackrel{9}{\mathrm{C}} \mathrm{H}_{2}-\stackrel{10}{-} \mathrm{CH}_{2}-\mathrm{CH}_{2}-\stackrel{12}{\mathrm{C}} \mathrm{H}_{2}-{ }^{\mathrm{C}} \mathrm{CH}_{2}-\mathrm{CH}_{3}
\end{aligned}
$$

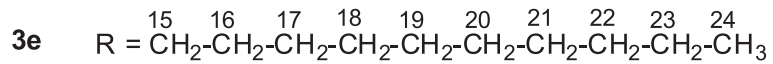<smiles>[R]OC(=O)C1=C(C)N(C)C(C)=C(C(=O)O[R])C1c1ccncc1</smiles>

$$
\begin{aligned}
& \text { 5a } \quad \mathrm{R}=\stackrel{7}{\mathrm{C}} \mathrm{H}_{2}-\stackrel{8}{\mathrm{C}} \mathrm{H}_{2}-\stackrel{9}{\mathrm{C} N} \quad \mathrm{R}^{\prime}=\stackrel{10}{\mathrm{C}} \mathrm{H}_{3} \\
& \text { 5b } \quad \mathrm{R}=\mathrm{R}^{\prime}=\stackrel{7}{\mathrm{C}} \mathrm{H}_{2}-\stackrel{8}{\mathrm{C}} \mathrm{H}_{2}-\stackrel{9}{\mathrm{C}} \mathrm{H}_{2}-\stackrel{10}{\mathrm{C}} \mathrm{H}_{2}-\stackrel{11}{\mathrm{C}} \mathrm{H}_{2}-\stackrel{12}{\mathrm{C}} \mathrm{H}_{2}-\stackrel{13}{\mathrm{C}} \mathrm{H}_{2}-\stackrel{14}{\mathrm{C}} \mathrm{H}_{3}
\end{aligned}
$$

Figure 1. Chemical structures for 1,4-dihydropyridine derivatives.

distinguished in compounds $\mathbf{1 d}, \mathbf{3 d}$ and $\mathbf{3 e}$, because of the similarity of the substituents on $\mathrm{C}-3$ and C-5. The ${ }^{1} \mathrm{H}$ NMR spectra of compounds $\mathbf{1}, \mathbf{2}, \mathbf{3}, \mathbf{4}$ and $\mathbf{5}$, also show signals between 7.0-8.5 ppm corresponding to the protons of the pyridine ring on $\mathrm{C}-4$, showing the characteristic multiplicity depending on the position of the nitrogen atom in the pyridine ring. In compounds $\mathbf{6 a}$ and $\mathbf{6 b}$ endowed with a $p$-nitrophenyl ring, the aromatic protons appear at 8.1 and 7.4 ppm as an AA'BB' system.<smiles>[R]OC(=O)C1=C(C)NC(C)=C(C(=O)OC)C1c1ccccn1</smiles>

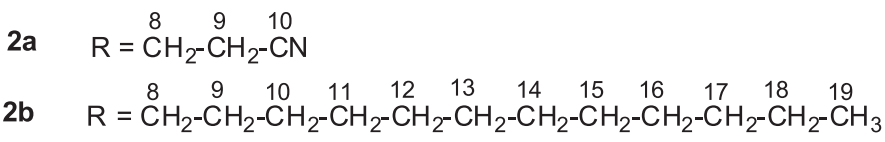<smiles>[R]OC(=O)C1=C(C)NC(C)=C(C(=O)OC)C1c1cccnc1</smiles>

4a $\quad \mathrm{R}=\stackrel{8}{\mathrm{C}} \mathrm{H}_{2}-\stackrel{9}{\mathrm{C}} \mathrm{H}_{2}-\stackrel{10}{\mathrm{CN}}$

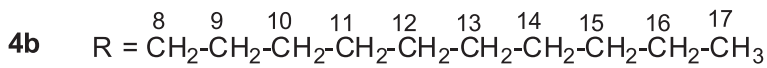

4c $\mathrm{R}=\stackrel{8}{\mathrm{C}} \mathrm{H}_{2}-\stackrel{9}{\mathrm{C}} \mathrm{H}_{2}-\stackrel{10}{\mathrm{O}}-\stackrel{11}{\mathrm{C}} \mathrm{H}_{2}-\stackrel{12}{-} \mathrm{CH}_{2}-\stackrel{13}{\mathrm{C}} \mathrm{H}_{2}-\stackrel{14}{\mathrm{C}} \mathrm{H}_{3}$<smiles>[R]OC(=O)C1=C(C)NC(C)=C(C(=O)OC)[C@H]1c1ccc([N+](=O)[O-])cc1</smiles>

$\begin{array}{ll}\text { 6a } & \mathrm{R}=\stackrel{8}{\mathrm{C}} \mathrm{H}_{2}-\stackrel{9}{\mathrm{C}} \mathrm{H}_{2}-\stackrel{10}{\mathrm{C} N} \\ \mathbf{6 b} & \mathrm{R}=\stackrel{8}{\mathrm{C}} \mathrm{H}_{2}-\stackrel{9}{\mathrm{C}} \mathrm{H}_{2}-\stackrel{10}{\mathrm{C}} \mathrm{H}_{2}-\stackrel{11}{\mathrm{C}} \mathrm{H}_{2}-\stackrel{12}{\mathrm{C}} \mathrm{H}_{2}-\stackrel{13}{\mathrm{C}} \mathrm{H}_{2}-\stackrel{14}{\mathrm{C}} \mathrm{H}_{2}-\stackrel{15}{\mathrm{C}} \mathrm{H}_{3}\end{array}$

The alkoxycarbonyl group shows a number of signals which depends of the size of the chain. In these cases, the methylene group joint to the oxygen atom of the alkoxycarbonyl chain $\left(-\mathrm{O}-\mathrm{CH}_{2}-\mathrm{R}\right)$ on $\mathrm{C}-3$ and $\mathrm{C}-5$, appears at $c a .3 .9 \mathrm{ppm}$ as a multiplet due to the presence of a stereogenic center on C-4. The rest of the aliphatic protons appear as a broad multiplet at $c a .1 .2 \mathrm{ppm}$ and terminal methyl group as a triplet at $\delta 0.8 \mathrm{ppm}$. The protons of the $\alpha$-methylene group corresponding to the 
cyanoethyl group in compounds $1 \mathbf{1 c}, \mathbf{2 a}, \mathbf{3 c}, \mathbf{4 a}, \mathbf{5 a}$ and 6a appear as a multiplet at $\delta 2.8 \mathrm{ppm}$ while the $\beta$-protons resonate at $4.1 \mathrm{ppm}$ forming a triplet.

The ${ }^{13} \mathrm{C}$ NMR spectra of these compounds exhibit signals in the carbonyl, aromatic and aliphatic regions. In order to assign unequivocally the signals corresponding to the heterocyclic ring, we used $1 \mathrm{D}$ and $2 \mathrm{D}$ techniques: DEPT(135), HMQC and HMBC.

For the 1,4-DHP ring, the spectra showed four quaternary carbon signals (C-2, C-3, C-5, and C-6) and one tertiary carbon signal (C-4). As can be seen in Table 2, the signals corresponding to the 1,4-DHP carbons ring are relatively insensitive to the nature of the substituents on $\mathrm{C}-3, \mathrm{C}-4$, and $\mathrm{C}-5$. The ${ }^{13} \mathrm{C}$ NMR spectra of these compounds show the signal for $\mathrm{C}-3$ and C-5 at lower $\delta$ values ( $c a .100 \mathrm{ppm}$ ) than those expected for typical olefinic carbons atoms, while C-2 and C-6 signals appear at higher $\delta$ values (ca.146 ppm) and C-4 appears between 36.8 and $41.3 \mathrm{ppm}$. These findings have been accounted for by the strong push-pull effect of the groups linked to the olefinic double bonds, similar to that previously observed in other related molecules. ${ }^{8-11}$ The alkoxycarbonyl $\mathrm{sp}^{2}$-carbon (COOR or $\mathrm{COOR}^{1}$ ) on C-3 and C-5 appears at 166-167 ppm. In compounds 1c, 2a, 3c, 4a, 5a and 6a the cyano group appears at $c a .119 \mathrm{ppm}$.

As we can see in Table 2, some carbons of the alkoxy chain do not present remarkable difficulties to be resolved and all nuclei were assigned on the basis of coupling constant and cross peaks on the 2D spectra. The rest of the signals are in agreement with the nature of aromatic or aliphatic carbon atoms. All the compounds showed a similar trend in the chemical shifts of the common moiety of the molecular backbone.

Also the ${ }^{15} \mathrm{~N}$ NMR spectrum of the compounds $\mathbf{2 a}, \mathbf{4 a}, \mathbf{4} \mathbf{c}$ and $\mathbf{5 a}$ were recorded in order to ensure the structure. The ${ }^{15} \mathrm{~N}$ HMQC, using two different values

Table 1. ${ }^{1} \mathrm{H}$ NMR data for compounds 1a-d, 2a-b, 3a-e, 5a-b, 6a-b $(\delta$, ppm; $J, \mathrm{~Hz})$

\begin{tabular}{|c|c|c|c|c|c|c|c|c|c|c|c|c|c|c|}
\hline Comp. & $\mathrm{N}-\mathrm{H}$ & H-4 & $\mathrm{CH}_{3}-\mathrm{C}-2$ & $\mathrm{CH}_{3}-\mathrm{C}-5$ & H-7 & H-8 & H-9 & $\mathrm{H}-10$ & H-11 & H-12 & $\mathrm{H}-13$ & H-14 & $\mathrm{H}-15$ & H-16 \\
\hline $1 a$ & $8.78, \mathrm{~s}$ & $5.01, \mathrm{~s}$ & $2.23, \mathrm{~s}$ & $2.22, \mathrm{~s}$ & $3.94, \mathrm{~m}$ & $1.48, \mathrm{~m}$ & & & 1.24 , br s & & & $\begin{array}{l}0.85, \mathrm{t} \\
(J 6.8)\end{array}$ & $3.53, \mathrm{~s}$ & - \\
\hline 1b & $8.76, \mathrm{~s}$ & $5.00, \mathrm{~s}$ & $2.23, \mathrm{~s}$ & $2.21, \mathrm{~s}$ & $3.90, \mathrm{~m}$ & $1.47, \mathrm{~m}$ & & & 1.20 , br s & & & $\begin{array}{l}0.84, \mathrm{t} \\
(J 6.8)\end{array}$ & $3.90, \mathrm{~m}$ & $\begin{array}{l}1.12, \mathrm{t} \\
(J 7.4)\end{array}$ \\
\hline 1c & $8.99, \mathrm{~s}$ & $5.02, \mathrm{~s}$ & $2.24, \mathrm{~s}$ & $2.23, \mathrm{~s}$ & $3.93, \mathrm{~m}$ & $1.48, \mathrm{~m}$ & & & 1.20 , br. s & & & $\begin{array}{l}0.85, \mathrm{t} \\
(J 6.7)\end{array}$ & $\begin{array}{l}4.13, \mathrm{t} \\
(J 5.8)\end{array}$ & $2.83, \mathrm{~m}$ \\
\hline 1d & $8.77, \mathrm{~s}$ & $5.02, \mathrm{~s}$ & \multicolumn{2}{|c|}{$2.22, \mathrm{~s}$} & $3.91, \mathrm{~m}$ & $1.48, \mathrm{~m}$ & & & 1.21 , br s & & & $\begin{array}{l}0.85, \mathrm{t} \\
(J 6.4)\end{array}$ & & \\
\hline $2 \mathrm{a}$ & $8.90, \mathrm{~s}$ & $5.01, \mathrm{~s}$ & $2.25, \mathrm{~s}$ & $2.24, \mathrm{~s}$ & $3.53, \mathrm{~s}$ & $\begin{array}{l}4.14, \mathrm{t} \\
(J 6.0)\end{array}$ & $2.83, \mathrm{~m}$ & - & - & - & - & - & - & - \\
\hline $2 b$ & $8.79, \mathrm{~s}$ & $5.01, \mathrm{~s}$ & $2.23, \mathrm{~s}$ & $2.21, \mathrm{~s}$ & $3.53, \mathrm{~s}$ & $3.91, \mathrm{~m}$ & $1.47, \mathrm{~m}$ & & & & 1.23, br s & & & \\
\hline 3a & $8.97, \mathrm{~s}$ & $4.89, \mathrm{~s}$ & $2.28, \mathrm{~s}$ & $2.26, \mathrm{~s}$ & $3.90, \mathrm{~m}$ & $1.48, \mathrm{~m}$ & & & 1.21 , br s & & & $\begin{array}{l}0.85, \mathrm{t} \\
(J 6.6)\end{array}$ & $3.54, \mathrm{~s}$ & - \\
\hline $3 b^{a}$ & $6.61, \mathrm{~s}$ & $4.98, \mathrm{~s}$ & $2.34, \mathrm{~s}$ & $2.32, \mathrm{~s}$ & $4.05, \mathrm{~m}$ & $1.58, \mathrm{~m}$ & & & 1.20 , br s & & & $\begin{array}{l}0.88, \mathrm{t} \\
(J \text { 6.7) }\end{array}$ & $4.05, \mathrm{~m}$ & $\begin{array}{l}1.19, \mathrm{t} \\
(J 7.1)\end{array}$ \\
\hline $3 c$ & $9.05, \mathrm{~s}$ & $4.86, \mathrm{~s}$ & $2.28, \mathrm{~s}$ & $2.27, \mathrm{~s}$ & $4.16, \mathrm{~m}$ & $1.48, \mathrm{~m}$ & & & $1.20 \mathrm{br} \mathrm{s}$ & & & $\begin{array}{l}0.85, \mathrm{t} \\
(J 6.9)\end{array}$ & $4.13, \mathrm{~m}$ & $2.84, \mathrm{~m}$ \\
\hline $3 d^{a}$ & 7.97, s & $4.75, \mathrm{~s}$ & \multicolumn{2}{|c|}{$2.10, \mathrm{~s}$} & $\begin{array}{l}3.36 \mathrm{t} \\
(J 6.5)\end{array}$ & $1.35, \mathrm{~m}$ & & & $1.02, \mathrm{~m}$ & & & $\begin{array}{l}0.64, \mathrm{t} \\
(J 6.5)\end{array}$ & - & - \\
\hline $3 e^{a}$ & $6.55, \mathrm{~s}$ & $5.03, \mathrm{~s}$ & \multicolumn{2}{|c|}{$2.36, \mathrm{~s}$} & $4.02, \mathrm{~m}$ & $1.58, \mathrm{~m}$ & & & $1.25, \mathrm{~m}$ & & & $\begin{array}{l}0.88, \mathrm{t} \\
(J 6.4)\end{array}$ & $4.02, \mathrm{~m}$ & $1.58, \mathrm{~m}$ \\
\hline $4 a$ & $9.08, \mathrm{~s}$ & $4.86, \mathrm{~s}$ & $2.29, \mathrm{~s}$ & $2.28, \mathrm{~s}$ & $3.54, \mathrm{~s}$ & $\begin{array}{l}4.15, \mathrm{t} \\
(J 5.9)\end{array}$ & $2.84, \mathrm{~m}$ & - & - & - & - & - & - & - \\
\hline $4 b$ & $8.98, \mathrm{~s}$ & $4.85, \mathrm{~s}$ & $2.27, \mathrm{~s}$ & $2.25, \mathrm{~s}$ & $3.54, \mathrm{~s}$ & $3.92, \mathrm{~m}$ & $1.47, \mathrm{~m}$ & & & & 1.22 , br. s & & & \\
\hline $4 c$ & $9.00, \mathrm{~s}$ & $4.86, \mathrm{~s}$ & $2.26, \mathrm{~s}$ & $2.21, \mathrm{~s}$ & $3.52, \mathrm{~s}$ & $4.06, \mathrm{~m}$ & $3.49, \mathrm{~m}$ & - & $3.35, \mathrm{~m}$ & $1.45, \mathrm{~m}$ & $1.30, \mathrm{~m}$ & $\begin{array}{l}0.86, \mathrm{t} \\
(J 6.91)\end{array}$ & - & - \\
\hline $5 a$ & $9.11, \mathrm{~s}$ & $4.88, \mathrm{~s}$ & $2.29, \mathrm{~s}$ & $2.28, \mathrm{~s}$ & $\begin{array}{l}4.16, \mathrm{t}, \\
(J 5.95)\end{array}$ & $2.84, \mathrm{~m}$ & - & $3.55, \mathrm{~s}$ & - & - & - & - & - & - \\
\hline $5 b$ & $8.99, \mathrm{~s}$ & $4.88, \mathrm{~s}$ & $2.27, \mathrm{~s}$ & $2.29, \mathrm{~s}$ & $3.94, \mathrm{~m}$ & $1.49, \mathrm{~m}$ & & & $1.21, \mathrm{~m}$ & & & $\begin{array}{l}0.85, \mathrm{t} \\
(J 6.46)\end{array}$ & & \\
\hline $6 a$ & $9.19, \mathrm{~s}$ & $4.98, \mathrm{~s}$ & $2.29, \mathrm{~s}$ & $2.28, \mathrm{~s}$ & $3.53, \mathrm{~s}$ & $\begin{array}{l}4.14, \mathrm{t} \\
(J 5.3)\end{array}$ & $2.83, \mathrm{~m}$ & - & - & - & - & - & - & - \\
\hline $6 b$ & $9.03, \mathrm{~s}$ & 4.97, s & $2.29, \mathrm{~s}$ & $2.25, \mathrm{~s}$ & $3.54, \mathrm{~s}$ & $3.97, \mathrm{~m}$ & & $1.44, \mathrm{~m}$ & & & 1.08, br s & & $\begin{array}{l}0.83, \mathrm{t} \\
(J 7.0)\end{array}$ & - \\
\hline
\end{tabular}


Table 1. Continuation

\begin{tabular}{|c|c|c|c|c|c|c|c|c|c|}
\hline Comp. & $\mathrm{H}-17 \quad \mathrm{H}$ & $\mathrm{H}-18$ & H-19 & $\mathrm{H}-20$ & $\mathrm{H}-21$ & $\mathrm{H}-22$ & $\mathrm{H}-23$ & $\mathrm{H}-24$ & Aromatics \\
\hline $1 \mathrm{a}$ & - & - & - & - & - & - & - & - & $8.38\left(1 \mathrm{H}, \mathrm{d}, J 4.7, \mathrm{H} 3{ }^{\prime}\right), 7.58\left(1 \mathrm{H}, \mathrm{td}, J 7.7, J 1.8, \mathrm{H} 5{ }^{\prime}\right), 7.11$ (2H, m, H4', H6') \\
\hline 1b & - & - & - & - & - & - & - & - & $\begin{array}{l}8.38\left(1 \mathrm{H}, \text { br d, J 4.5, H3'), } 7.58\left(1 \mathrm{H}, \mathrm{td}, J 7.6, J 1.9, \mathrm{H} 5^{\prime}\right), 7.12(1 \mathrm{H}, \mathrm{d}, \mathrm{H} 6 \text { ', }\right. \\
\text { overlaping with } 7.09), 7.09\left(1 \mathrm{H}, \mathrm{d}, \mathrm{H} 4^{\prime} \text {, overlaping with } 7.12\right)\end{array}$ \\
\hline $1 \mathbf{c}$ & - & - & - & - & - & - & - & - & $\begin{array}{l}8.39\left(1 \mathrm{H}, \text { br. d, } J 4.8, \mathrm{H} 3^{\prime}\right), 7.56\left(1 \mathrm{H}, \mathrm{td}, J 7.8, J 1.6, \mathrm{H} 5^{\prime}\right), 7.19(1 \mathrm{H}, \mathrm{d}, J 7.8 \text {, } \\
\left.\mathrm{H} 6^{\prime}\right), 7.09\left(1 \mathrm{H}, \mathrm{dd}, J 7.8, J 4.8, \mathrm{H}^{\prime}\right)\end{array}$ \\
\hline 1d & - & - & - & - & - & - & - & - & $8.38\left(1 \mathrm{H}, \mathrm{d}, J 4.0, \mathrm{H}^{\prime}\right), 7.55\left(1 \mathrm{H}, \mathrm{td}, J 7.6, J 1.8, \mathrm{H} 5^{\prime}\right), 7.10\left(2 \mathrm{H}, \mathrm{m}, \mathrm{H} 4{ }^{\prime}, \mathrm{H} 6^{\prime}\right)$ \\
\hline $2 \mathbf{a}$ & - & - & - & - & - & - & - & & $\begin{array}{l}8.40\left(1 \mathrm{H}, \mathrm{d}, J 4.7, \mathrm{H}^{\prime}\right), 7.58\left(1 \mathrm{H}, \mathrm{td}, J 7.7, J 1.8, \mathrm{H} 5^{\prime}\right), 7.18\left(1 \mathrm{H}, \mathrm{d}, J 7.7, \mathrm{H} 6^{\prime}\right), \\
7.11\left(1 \mathrm{H}, \mathrm{dd}, J 7.7, J 4.7, \mathrm{H}^{\prime}\right)\end{array}$ \\
\hline $2 \mathbf{b}$ & $1.23,1$ & & $\begin{array}{l}0.85, \mathrm{t} \\
(J 6.4)\end{array}$ & - & - & - & - & - & $8.38\left(1 \mathrm{H}, \mathrm{d}, J 4.0, \mathrm{H}^{\prime}\right), 7.59$ (1H, td, J7.6, J1.8, H5'), 7.09 (2H, m, H4', H6') \\
\hline $3 \mathbf{a}$ & - & - & - & - & - & - & - & - & $\begin{array}{l}8.35\left(1 \mathrm{H}, \mathrm{d}, J 1.6, \mathrm{H} 2^{\prime}\right), 8.31\left(1 \mathrm{H}, \mathrm{dd}, J 4.7, J 1.6, \mathrm{H}^{\prime}\right), 7.47(1 \mathrm{H}, \mathrm{dt}, J 7.8, \\
\left.J 1.6, \mathrm{H} 6^{\prime}\right), 7.23\left(1 \mathrm{H}, \mathrm{dd}, J 7.8, J 4.7, \mathrm{H} 5^{\prime}\right)\end{array}$ \\
\hline $3 \mathbf{b}^{\mathrm{a}}$ & - & - & - & - & - & - & - & - & $\begin{array}{l}8.52\left(1 \mathrm{H}, \mathrm{d}, J 1.6, \mathrm{H}^{\prime}\right), 8.36\left(1 \mathrm{H}, \mathrm{dd}, J 4.8, J 1.6, \mathrm{H}^{\prime}\right), 7.61(1 \mathrm{H}, \mathrm{dt}, J 7.8 \text {, } \\
\left.J 1.6, \mathrm{H} 6^{\prime}\right), 7.15\left(1 \mathrm{H}, \mathrm{dd}, J 7.8, J 4.8, \mathrm{H} 5^{\prime}\right)\end{array}$ \\
\hline $3 \mathbf{c}$ & - & - & - & - & - & - & - & - & $\begin{array}{l}8.39\left(1 \mathrm{H}, \mathrm{d}, J 1.6, \mathrm{H} 2^{\prime}\right), 8.30\left(1 \mathrm{H}, \mathrm{dd}, J 4.7, J 1.6, \mathrm{H}^{\prime}\right), 7.53(1 \mathrm{H}, \mathrm{dt}, J 7.8 \text {, } \\
\left.J 1.6, \mathrm{H} 6^{\prime}\right), 7.23\left(1 \mathrm{H}, \mathrm{dd}, J 7.8, J 4.7, \mathrm{H} 5^{\prime}\right)\end{array}$ \\
\hline $3 \mathbf{d}^{\mathrm{a}}$ & & & & $1.02, \mathrm{~m}$ & & & & $\begin{array}{l}0.85, \mathrm{t} \\
(J 6.9)\end{array}$ & $\begin{array}{l}8.26\left(1 \mathrm{H}, \mathrm{d}, J 1.8, \mathrm{H}^{\prime}\right), 8.10\left(1 \mathrm{H}, \mathrm{dd}, J 5.0, J 1.8, \mathrm{H}^{\prime}\right) 7.43(1 \mathrm{H}, \mathrm{dt}, J 7.0 \text {, } \\
\left.J 1.8, \mathrm{H} 6^{\prime}\right), 6.98\left(1 \mathrm{H}, \mathrm{dd}, J 7.0, J 5.0, \mathrm{H}^{\prime}\right)\end{array}$ \\
\hline $3 \mathrm{e}^{\mathrm{a}}$ & - & - & - & - & - & - & - & - & $\begin{array}{l}8.51\left(1 \mathrm{H}, \mathrm{d}, J 1.8, \mathrm{H} 2^{\prime}\right), 8.38\left(1 \mathrm{H}, \mathrm{dd}, J 5.0, J 1.8, \mathrm{H}^{\prime}\right) 7.83(1 \mathrm{H}, \mathrm{dt}, J 7.8 \text {, } \\
\left.J 1.8, \mathrm{H} 6^{\prime}\right), 7.30\left(1 \mathrm{H}, \mathrm{dd}, J 7.8, J 5.0, \mathrm{H} 5^{\prime}\right)\end{array}$ \\
\hline $4 a$ & - & - & - & - & - & - & - & - & $\begin{array}{l}8.38\left(1 \mathrm{H}, \mathrm{d}, J 1.7, \mathrm{H} 2^{\prime}\right), 8.32\left(1 \mathrm{H}, \mathrm{dd}, J 4.7, J 1.7, \mathrm{H}^{\prime}\right), 7.52(1 \mathrm{H}, \mathrm{dt}, J 7.7, \\
\left.J 1.7, \mathrm{H} 6^{\prime}\right), 7.24\left(1 \mathrm{H}, \mathrm{dd}, J 7.7, J 4.7, \mathrm{H} 5^{\prime}\right)\end{array}$ \\
\hline $4 b$ & $\begin{array}{l}0.85, \mathrm{t} \\
(J 6.4)\end{array}$ & - & - & - & - & - & - & - & $\begin{array}{l}8.35\left(1 \mathrm{H}, \mathrm{d}, J 1.6, \mathrm{H} 2^{\prime}\right), 8.30\left(1 \mathrm{H}, \mathrm{dd}, J 4.7, J 1.6, \mathrm{H}^{\prime}\right), 7.48(1 \mathrm{H}, \mathrm{dt}, J 7.9, \\
\left.J 1.6, \mathrm{H} 6^{\prime}\right), 7.24\left(1 \mathrm{H}, \mathrm{dd}, J 7.9, J 4.7, \mathrm{H} 5^{\prime}\right)\end{array}$ \\
\hline $4 c$ & - & - & - & - & - & - & - & - & $\begin{array}{l}8.38\left(1 \mathrm{H}, \mathrm{d}, J 1.7, \mathrm{H} 2^{\prime}\right), 8.31\left(1 \mathrm{H}, \mathrm{dd}, J 4.7, J 1.7, \mathrm{H}^{\prime}\right), 7.50(1 \mathrm{H}, \mathrm{dt}, J 7.9, \\
\left.J 1.7, \mathrm{H} 6^{\prime}\right), 7.23\left(1 \mathrm{H}, \mathrm{dd}, J 7.9, J 4.7, \mathrm{H} 5^{\prime}\right)\end{array}$ \\
\hline $5 \mathbf{a}$ & - & - & - & - & - & - & - & - & 8.40 (2H, d, J 5.9, H3', H5'), 7.14 (2H, d, J 5.95, H2', H6') \\
\hline $5 \mathbf{b}$ & - & - & - & - & - & - & - & - & $8.38\left(2 \mathrm{H}, \mathrm{d}, J 5.95, \mathrm{H} 3^{\prime}, \mathrm{H} 5^{\prime}\right), 7.10(2 \mathrm{H}, \mathrm{d}, J$ 5.9, H2', H6') \\
\hline $6 \mathbf{a}$ & - & - & - & - & - & - & - & - & 8.09 (2H, d, J 8.7, H3', H5'), 7.44 (2H, d, J 8.7, H2', H6') \\
\hline $6 \mathbf{b}$ & - & - & - & - & - & - & - & - & $8.10(2 \mathrm{H}, \mathrm{d}, J$ 8.7, H3', H5'), 7.40 (2H, d, J 8.7, H2', H6') \\
\hline
\end{tabular}

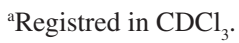

of the $\mathrm{N}, \mathrm{H}$ coupling constants, allow us to determine the chemical shift of the nitrogen atoms present in the molecule. In the HMQC, using a value of ${ }^{1} J(\mathrm{~N}, \mathrm{H}) 80 \mathrm{~Hz}$, the hydrogen signal correlates with the $\mathrm{N}-1$ atom at ca. $-239 \mathrm{ppm}$. However in the HMQC using values of ${ }^{\mathrm{n}} J(\mathrm{~N}, \mathrm{H}) 5 \mathrm{~Hz}$ the hydrogen signals showing correlation were those bonded through more than one bond. This confirms the position of $\mathrm{N}-1$ and allows us to assign the position of the pyridine nitrogen at $c a .-66 \mathrm{ppm}$, which is in agreement with values reported in the literature ${ }^{12}$ (see Table 3).

\section{Experimental}

\section{Material}

Compounds 1, 2, 3, 4, 5 and $\mathbf{6}$ were prepared according to the synthetic method described previously. ${ }^{6}$ The $1,4-$ DHPs derivatives were purified by recrystallization. Compatible IR and mass spectra and combustion analyses were taken for each compound.

\section{Spectra}

All NMR experiments were performed at $298 \mathrm{~K}$ for a solution of $30 \mathrm{mg}$ of compound dissolved in $0.7 \mathrm{~mL}$ of DMSO- $d_{6}$ on a Bruker AVANCE-300 instrument with a 5-mm QNP probe head equipped with shielded Z-gradient coil. ${ }^{1} \mathrm{H}$ NMR spectra were recorded at a proton frequency of $300.13 \mathrm{MHz}$ with a spectral width of $4.5 \mathrm{kHz}$ and a $2.15 \mu \mathrm{s}\left(30^{\circ}\right)$ pulse. The acquisition time was $1.8 \mathrm{~s}$ and relaxation delay $1 \mathrm{~s} ; 16$ scans with $16 \mathrm{k}$ data points each were used. The ${ }^{13} \mathrm{C}$ NMR were obtained using a spectral width of $20 \mathrm{kHz}$, a $1.9 \mu \mathrm{s}\left(30^{\circ}\right)$ pulse, and a $1.7 \mathrm{~s}$ acquisition time; 512 scans with $64 \mathrm{k}$ data points each were used. Exponential multiplication was applied before Fourier transformation in both cases. The chemical shifts were referenced to DMSO- $d_{6}$. The one-bond heteronuclear correlation (HMQC) spectra were obtained using the inv4gs program in the Bruker software. The spectra resulted from a $256 \times 2048$ data matrix with 8 scans per $\mathrm{t}_{1}$ increment. Spectral widths of $3.5 \mathrm{kHz}$ in $\mathrm{f}_{2}$ and $16.0 \mathrm{kHz}$ in $\mathrm{f}_{1}$ were used. The acquisition time was $0.30 \mathrm{~s}$, the delay was set to 
$3.45 \mathrm{~ms}$, (corresponding to an average ${ }^{1} J(\mathrm{C}, \mathrm{H})$ of $145 \mathrm{~Hz}$ ), and the recycle time was $1.44 \mathrm{~s}$. Fourier transformation was done on a $2 \mathrm{k} \times 1 \mathrm{k}$ data matrix. The long-range ${ }^{1} \mathrm{H}-{ }^{13} \mathrm{C}$ correlation (HMBC) spectra were obtained using the inv4gslplrnd program in the Bruker software. The spectra resulted from a $256 \times 2048$ data matrix with 16 scans per $\mathrm{t}_{1}$ increment. A spectral width of $3.5 \mathrm{kHz}$ in $\mathrm{f}_{2}$ and $16.7 \mathrm{kHz}$ in $\mathrm{f} 1$ were used. The acquisition time was $0.30 \mathrm{~s}$, the delays were set to $3.45 \mathrm{~ms}\left(1 / 2 J_{\mathrm{C}, \mathrm{H}}\right)$ and $65 \mathrm{~ms}$ (corresponding to an average $1 /{ }^{\mathrm{n}} J_{C, H}$ of $7.7 \mathrm{~Hz}$ ), and the recycle time was $1.44 \mathrm{~s}$. Fourier transformation was done on a $2 \mathrm{k} \times 1 \mathrm{k}$ data matrix.
${ }^{15} \mathrm{~N}$ NMR spectra were recorded at $50.687 \mathrm{MHz}$ on a Bruker Avance-500 instrument. The chemical shifts were referenced to $\mathrm{CH}_{3} \mathrm{NO}_{2}$ at $0.00 \mathrm{ppm}$. The one-bond heteronuclear correlation (HMQC) was obtained using the inv4gpqf program in the Bruker software. The spectra resulted from a $128 \times 1024$ data matrix with 32 scans per $\mathrm{t}_{1}$ increment. Spectral widths of $6.5 \mathrm{kHz}$ in $\mathrm{f}_{2}$ and $20.5 \mathrm{kHz}$ in $\mathrm{f}_{1}$ were used. The acquisition time was $0.1 \mathrm{~s}$ and delay was set to $6 \mathrm{~ms}$ (corresponding to ${ }^{1} J(\mathrm{~N}, \mathrm{H})$ of $80 \mathrm{~Hz}$ ) and $100 \mathrm{~ms}$ for ${ }^{\mathrm{n}} \mathrm{J}(\mathrm{N}, \mathrm{H})$ of $5 \mathrm{~Hz}$ using a recycle time of $2 \mathrm{~s}$.

Table 2. ${ }^{13} \mathrm{C}$ NMR data for compounds 1a-d, 2a-b, 3a-e, 5a-b, 6a-b $(\delta, \mathrm{ppm})$

\begin{tabular}{|c|c|c|c|c|c|c|c|c|c|c|c|}
\hline & Aromatics & $(\mathrm{C}-5-\underline{\mathrm{C}}=\mathrm{O})$ & $(\mathrm{C}-3-\mathrm{C}=\mathrm{O})$ & $\mathrm{C}-2$ & C-3 & C-4 & $\mathrm{C}-5$ & C-6 & $\mathrm{C}-7$ & C-8 & C-9 \\
\hline $1 \mathbf{a}$ & $\begin{array}{c}164.9\left(\mathrm{C}^{\prime}\right), 149.1\left(\mathrm{C}^{\prime}{ }^{\prime}\right) \\
135.6(\mathrm{C} 5 '), 121.4 \\
121.3\left(\mathrm{C}^{\prime}, \mathrm{C} 6^{\prime}\right)\end{array}$ & 167.5 & 166.9 & $\begin{array}{l}146.1^{\mathrm{b}} \\
146.0^{\mathrm{b}}\end{array}$ & 100.3 & 41.2 & 100.2 & $\begin{array}{l}146.1^{\mathrm{b}} \\
146.0^{\mathrm{b}}\end{array}$ & 62.9 & 28.3 & 25.5 \\
\hline $1 \mathrm{~b}$ & $\begin{array}{l}165.1\left(\mathrm{C}^{\prime}{ }^{\prime}\right), 135.4\left(\mathrm{C}^{\prime}\right), 121.7 \\
\left(\mathrm{C}^{\prime}\right), 121.3\left(\mathrm{C}^{\prime}\right), 149.1\left(\mathrm{C} 3^{\prime}\right)\end{array}$ & 166.9 & 166.9 & $\begin{array}{l}146.2^{\mathrm{b}} \\
145.9^{\mathrm{b}}\end{array}$ & $\begin{array}{l}100.4^{\mathrm{b}} \\
100.3^{\mathrm{b}}\end{array}$ & 41.3 & $\begin{array}{l}100.4^{\mathrm{b}} \\
100.3^{\mathrm{b}}\end{array}$ & $\begin{array}{l}146.2^{\mathrm{b}} \\
145.9^{\mathrm{b}}\end{array}$ & 62.9 & 28.3 & 25.6 \\
\hline $1 \mathrm{c}$ & $\begin{array}{l}164.8\left(\mathrm{C}^{\prime}{ }^{\prime}\right), 149.1 \text { (C3') }^{\prime} 135.5 \\
\left(\mathrm{C}^{\prime}\right), 121.8 \text { (C6'), } 121.4\left(\mathrm{C}^{\prime}\right)\end{array}$ & 166.9 & 166.5 & $\begin{array}{l}147.1^{\mathrm{b}} \\
146.0^{\mathrm{b}}\end{array}$ & 100.7 & 41.1 & 99.5 & $\begin{array}{l}147.1^{\mathrm{b}} \\
146.0^{\mathrm{b}}\end{array}$ & 63.0 & 28.2 & 25.5 \\
\hline 1d & $\begin{array}{c}165.1\left(\mathrm{C}^{\prime} 1^{\prime}\right), 149.1\left(\mathrm{C}^{\prime}\right), 121.6, \\
121.3 \text { (C4', C6'), } 135.3 \text { (C5') }\end{array}$ & & 6.9 & 146.1 & 100.3 & 41.2 & 100.3 & 146.1 & 62.9 & 28.3 & 25.6 \\
\hline $2 a$ & $\begin{array}{l}164.7\left(\mathrm{C}^{\prime}\right), 149.1\left(\mathrm{C}^{\prime}\right) \\
121.4\left(\mathrm{C} 4^{\prime}\right), 135.8\left(\mathrm{C5}^{\prime}\right)\end{array}$ & 167.4 & 166.5 & $\begin{array}{l}147.2^{\mathrm{b}} \\
146.1^{\mathrm{b}}\end{array}$ & 99.5 & 41.0 & 100.5 & $\begin{array}{l}147.2^{\mathrm{b}} \\
146.1^{\mathrm{b}}\end{array}$ & 50.6 & 58.4 & 17.5 \\
\hline $2 b$ & $\begin{array}{c}164.9\left(\mathrm{C} 1^{\prime}\right), 149.1\left(\mathrm{C}^{\prime}\right) \\
135.5\left(\mathrm{C}^{\prime}\right), 121.4 \\
121.2\left(\mathrm{C}^{\prime}, \mathrm{C} 6\right. \\
\end{array}$ & 167.4 & 166.9 & $\begin{array}{l}146.1^{\mathrm{b}} \\
146.0^{\mathrm{b}}\end{array}$ & 100.3 & 41.1 & 100.2 & $\begin{array}{l}146.1^{\mathrm{b}} \\
146.0^{\mathrm{b}}\end{array}$ & 50.0 & 62.9 & 28.2 \\
\hline $3 \mathbf{a}$ & $\begin{array}{l}148.5\left(\mathrm{C} 2^{\prime}\right), 147.2\left(\mathrm{C} 4^{\prime}\right), 143.2 \\
\left(\mathrm{C} 1^{\prime}\right), 134.6 \text { (C6'), } 123.5\left(\mathrm{C} 5^{\prime}\right)\end{array}$ & 167.1 & 166.6 & 146.3 & $\begin{array}{l}100.9^{\mathrm{b}} \\
100.8^{\mathrm{b}}\end{array}$ & 36.8 & $\begin{array}{l}100.9^{\mathrm{b}} \\
100.8^{\mathrm{b}}\end{array}$ & 146.3 & 63.2 & 28.2 & 25.5 \\
\hline $3 \mathbf{b}^{\mathrm{a}}$ & $\begin{array}{l}149.4(\mathrm{C} 2 '), 147.1\left(\mathrm{C} 4^{\prime}\right), 143.5 \\
\left(\mathrm{C} 1^{\prime}\right), 135.6\left(\mathrm{C} 6 \text { '), } 123.0\left(\mathrm{C} 5^{\prime}\right)\right.\end{array}$ & $\begin{array}{l}167.3^{\mathrm{b}} \\
167.2^{\mathrm{b}}\end{array}$ & $\begin{array}{l}167.3^{\mathrm{b}} \\
167.2^{\mathrm{b}}\end{array}$ & $\begin{array}{l}145.0^{\mathrm{b}} \\
144.9^{\mathrm{b}}\end{array}$ & 103.1 & 37.7 & 103.1 & $\begin{array}{l}145.0^{\mathrm{b}} \\
144.9^{\mathrm{b}}\end{array}$ & 64.0 & 28.6 & 26.0 \\
\hline $3 c$ & $\begin{array}{l}148.6\left(\mathrm{C} 2^{\prime}\right), 147.2\left(\mathrm{C} 4^{\prime}\right), 143.1 \\
\left(\mathrm{C} 1^{\prime}\right), 123.4\left(\mathrm{C} 5^{\prime}\right), 134.8\left(\mathrm{C}^{\prime}\right)\end{array}$ & 166.1 & 166.5 & $\begin{array}{l}147.2^{\mathrm{b}} \\
146.1^{\mathrm{b}}\end{array}$ & $\begin{array}{l}101.4^{\mathrm{b}} \\
100.3^{\mathrm{b}}\end{array}$ & 36.8 & $\begin{array}{l}101.4^{\mathrm{b}} \\
100.3^{\mathrm{b}}\end{array}$ & $\begin{array}{c}147.2^{\mathrm{b}} \\
146.1\end{array}$ & 63.2 & 28.2 & 25.5 \\
\hline $3 d^{a}$ & $\begin{array}{l}147.7\left(\mathrm{C} 2^{\prime}\right), 145.3\left(\mathrm{C} 4{ }^{\prime}\right), 143.7 \\
\left(\mathrm{C} 1^{\prime}\right), 135.9 \text { (C6'), } 122.8\left(\mathrm{C}^{\prime}\right)\end{array}$ & 166.8 & 166.8 & 145.8 & 101.3 & 37.2 & 101.3 & 145.8 & 63.2 & 28.0 & 25.4 \\
\hline $3 \mathrm{e}^{\mathrm{a}}$ & $\begin{array}{c}\text { 144.7, } 144.6\left(\mathrm{C}^{\prime}, \mathrm{C}^{\prime}\right), 147.3\left(\mathrm{C} 2^{\prime}\right), \\
138.1\left(\mathrm{C}^{\prime}\right), 123.8\left(\mathrm{C}^{\prime}\right)\end{array}$ & 167.6 & 167.6 & 145.5 & 103.2 & 38.0 & 103.2 & 145.5 & 64.5 & 28.6 & 26.0 \\
\hline $4 a$ & $\begin{array}{l}148.5\left(\mathrm{C} 2^{\prime}\right), 147.3\left(\mathrm{C} 4^{\prime}\right), 142.9 \\
\left(\mathrm{C} 1^{\prime}\right), 134.7 \text { (C6'), } 123.5\left(\mathrm{C} 5^{\prime}\right)\end{array}$ & 166.9 & 166.1 & $\begin{array}{l}146.3^{\mathrm{b}} \\
146.2^{\mathrm{b}}\end{array}$ & 100.2 & 36.7 & 101.2 & $\begin{array}{l}146.3^{\mathrm{b}} \\
146.2^{\mathrm{b}}\end{array}$ & 50.8 & 58.6 & 17.4 \\
\hline $4 b$ & $\begin{array}{l}148.5\left(\mathrm{C} 2^{\prime}\right), 147.1\left(\mathrm{C} 4^{\prime}\right), 143.1 \\
\left(\mathrm{C} 1^{\prime}\right), 134.6 \text { (C6'), } 123.5\left(\mathrm{C}^{\prime}\right)\end{array}$ & 167.1 & 166.5 & $\begin{array}{l}146.3^{\mathrm{b}} \\
146.2^{\mathrm{b}}\end{array}$ & 100.8 & 37.2 & 100.9 & $\begin{array}{l}146.3^{\mathrm{b}} \\
146.2^{\mathrm{b}}\end{array}$ & 50.7 & 63.1 & 28.3 \\
\hline $4 c$ & $\begin{array}{l}148.8\left(\mathrm{C} 2^{\prime}\right), 147.3 \text { (C4'), } 143.0 \\
\left(\mathrm{C} 1^{\prime}\right), 134.9 \text { (C6'), } 123.7\left(\mathrm{C}^{\prime}\right)\end{array}$ & 166.5 & 167.0 & 147.7 & 101.2 & 36.9 & 101.0 & 147.7 & 50.8 & 68.3 & 62.8 \\
\hline $5 a$ & $\begin{array}{c}155.8\left(\mathrm{C} 1^{\prime}\right), 149.8\left(\mathrm{C}^{\prime}, \mathrm{C}^{\prime}\right), \\
122.7\left(\mathrm{C} 2^{\prime}, \mathrm{C}^{\prime}\right)\end{array}$ & 167.4 & 166.5 & $\begin{array}{l}147.1^{\mathrm{b}} \\
146.9^{\mathrm{b}}\end{array}$ & 100.9 & 38.6 & 100.5 & $\begin{array}{l}147.1^{\mathrm{b}} \\
146.9^{\mathrm{b}}\end{array}$ & 58.9 & 17.7 & - \\
\hline $5 \mathbf{b}$ & $\begin{array}{c}157.0\left(\mathrm{C} 1^{\prime}\right), 149.5\left(\mathrm{C}^{\prime}, \mathrm{C}^{\prime}\right) \\
122.5\left(\mathrm{C} 2^{\prime}, \mathrm{C} 6^{\prime}\right)\end{array}$ & 167.5 & 167.5 & 147.6 & 101.3 & 38.6 & 101.3 & 147.6 & 63.5 & 28.6 & 25.4 \\
\hline $6 a$ & $\begin{array}{c}155.2\left(\mathrm{C}^{\prime}{ }^{\prime}\right), 147.7\left(\mathrm{C}^{\prime}{ }^{\prime}\right) \\
128.8\left(\mathrm{C} 2^{\prime}, \mathrm{C}^{\prime}\right), 123.8\left(\mathrm{C} 3^{\prime}, \mathrm{C}^{\prime}\right)\end{array}$ & 167.0 & 166.2 & $\begin{array}{l}146.5^{\mathrm{b}} \\
146.0^{\mathrm{b}}\end{array}$ & 100.1 & 39.8 & 100.1 & $\begin{array}{l}146.5^{\mathrm{b}} \\
146.0^{\mathrm{b}}\end{array}$ & 51.2 & 58.9 & 17.7 \\
\hline $6 \mathrm{~b}$ & $\begin{array}{c}155.7\left(\mathrm{C} 4^{\prime}\right), 145.7\left(\mathrm{C}^{\prime}{ }^{\prime}\right), \\
128.7 \text { (C2', C6'), } 123.7 \text { (C3', C5') }\end{array}$ & 167.0 & 166.5 & $\begin{array}{l}146.6^{\mathrm{b}} \\
146.4^{\mathrm{b}}\end{array}$ & $\begin{array}{l}100.7^{\mathrm{b}} \\
100.6^{\mathrm{b}}\end{array}$ & 39.6 & $\begin{array}{l}100.7^{\mathrm{b}} \\
100.6^{\mathrm{b}}\end{array}$ & $\begin{array}{l}146.6^{\mathrm{b}} \\
146.4^{\mathrm{b}}\end{array}$ & 51.2 & 63.6 & 28.2 \\
\hline
\end{tabular}


Table 2. Continuation

\begin{tabular}{|c|c|c|c|c|c|c|c|c|c|c|c|c|c|c|c|c|c|c|}
\hline & $\mathrm{C}-10$ & $\mathrm{C}-11$ & $\mathrm{C}-12$ & $\mathrm{C}-13$ & $\mathrm{C}-14$ & $\mathrm{C}-15$ & $\mathrm{C}-16$ & $\mathrm{C}-17$ & C-18 & C-19 & $\mathrm{C}-20$ & $\mathrm{C}-21$ & $\mathrm{C}-22$ & $\mathrm{C}-23$ & $\mathrm{C}-24$ & $\mathrm{CH}_{3}-\mathrm{C}-6$ & $\mathrm{CH}_{3}-\mathrm{C}-2$ & $\mathrm{CN}$ \\
\hline $1 \mathbf{a}$ & 31.2 & 28.6 & 28.6 & 22.1 & 13.9 & 50.6 & - & - & - & - & - & - & - & - & - & \multicolumn{2}{|c|}{18.3} & - \\
\hline $1 \mathrm{~b}$ & 31.2 & 28.6 & 28.6 & 22.1 & 13.9 & 58.9 & 14.2 & - & & - & - & - & - & - & - & \multicolumn{2}{|c|}{18.2} & - \\
\hline $1 \mathrm{c}$ & 31.2 & 28.6 & 28.6 & 22.1 & 13.9 & 58.4 & 17.5 & - & - & - & - & - & - & - & - & 18.3 & 18.6 & 118.8 \\
\hline 1d & 31.2 & 28.6 & 28.6 & 22.1 & 13.9 & 62.9 & 28.3 & 25.6 & 31.2 & 28.6 & 28.6 & 22.1 & 13.9 & - & - & 18.3 & 18.6 & - \\
\hline $2 a$ & - & - & - & - & - & - & - & - & - & - & - & - & - & - & - & 18.3 & 18.6 & 118.8 \\
\hline $2 b$ & 31.3 & 29.0 & 29.0 & 28.9 & 28.7 & 28.6 & 28.6 & 25.5 & 22.1 & 13.9 & - & - & - & - & - & \multicolumn{2}{|c|}{18.3} & - \\
\hline $3 \mathbf{a}$ & 31.1 & 28.6 & 28.6 & 22.1 & 13.9 & 50.8 & - & - & - & - & - & - & - & - & - & 18.2 & 18.1 & - \\
\hline $3 b^{\mathrm{a}}$ & 31.7 & 29.2 & 29.2 & 22.6 & 14.1 & 59.8 & & - & - & - & - & - & - & - & - & \multicolumn{2}{|c|}{19.3} & - \\
\hline $3 c$ & 31.2 & 28.6 & 28.6 & 22.1 & 13.9 & 58.6 & 17.4 & - & - & - & - & - & - & - & - & 18.1 & 18.4 & 118.6 \\
\hline $3 \mathbf{d}^{\mathrm{a}}$ & 31.1 & 28.6 & 28.5 & 21.9 & 13.5 & - & - & - & - & - & - & - & - & - & - & \multicolumn{2}{|c|}{18.2} & - \\
\hline $3 \mathrm{e}^{\mathrm{a}}$ & 31.9 & 29.5 & 29.3 & 22.7 & 14.4 & 64.5 & 28.6 & 26.0 & 29.5 & 23.3 & 29.2 & 29.3 & 31.8 & 22.6 & 14.2 & 19.5 & 19.5 & - \\
\hline $4 a$ & - & - & - & - & - & - & - & - & - & - & - & - & & & & 18.2 & 18.5 & 118.7 \\
\hline $4 b$ & 25.9 & 31.6 & 29.2 & 29.2 & 29.0 & 28.9 & 22.5 & 13.0 & - & - & & - & - & - & - & 18.1 & 18.2 & - \\
\hline $4 c$ & - & 70.1 & 31.5 & 19.4 & 14.1 & - & - & - & - & - & - & - & - & - & - & \multicolumn{2}{|c|}{18.5} & - \\
\hline $5 a$ & 51.2 & - & - & - & - & - & - & - & - & - & - & - & - & - & - & 18.6 & 18.5 & 119.9 \\
\hline $5 b$ & 31.6 & 28.9 & 28.9 & 22.4 & 14.1 & & & & & & & & & & & \multicolumn{2}{|c|}{18.2} & - \\
\hline $6 a$ & - & - & - & - & - & - & - & - & - & - & - & - & - & - & - & 18.6 & 18.8 & 119.9 \\
\hline $6 \mathrm{~b}$ & 25.5 & 31.5 & 28.7 & 28.6 & 22.1 & 13.9 & & & & & & & & & & 18.3 & 18.2 & - \\
\hline
\end{tabular}

${ }^{\mathrm{a}}$ Registred in $\mathrm{CDCl}_{3}$; ${ }^{\text {bInterchangeable. }}$

Table 3. ${ }^{15} \mathrm{~N}$ HMQC data for 1,4-dihydropyridine derivatives (ppm)

\begin{tabular}{lcccc}
\hline Compound & $\mathrm{N}-1$ & $\mathrm{~N}-2^{\prime}$ & $\mathrm{N}-3^{\prime}$ & $\mathrm{N}-4^{\prime}$ \\
\hline $\mathbf{2 a}$ & -238.28 & -65.15 & - & - \\
$\mathbf{4 a}$ & -239.44 & - & -63.22 & - \\
$\mathbf{4 c}$ & -240.17 & - & -62.53 & - \\
$\mathbf{5 a}$ & -238.88 & - & - & -67.01 \\
\hline
\end{tabular}

\section{Conclusions}

We have recorded the ${ }^{1} \mathrm{H}$ and ${ }^{13} \mathrm{C}$ NMR spectra of several substituted 1,4-dihydropyridines (1,4-DHPs) endowed with long and functionalized chains in order to assign unequivocally all the chemical shifts. These spectral data are of interest to report the effect of the substitution pattern on the chemical shifts and to complete reference data previously reported. The 1,4-DHPs carbons ring are practically insensitive to the nature of substituents. Also, we present the ${ }^{15} \mathrm{~N}$ NMR spectroscopic data of the reported molecules.

\section{References}

1. Goldmann, S.; Stoltefuss, J.; Angew. Chem., Int. Ed. 1991, 30, 1559; Richter, M.; Molnar, J.; Hilgeroth, A.; J. Med. Chem. 2006, 49, 2838.

2. Jain, P.; Narang, G.; Jindal, D. P.; Bansal, R.; Calle, C.; Carron, R.; Pemberton, K.; Harvey, A. L.; Pharmazie 2006, 61, 400; Evdokimov, N. M.; Magedov , I. V.; Kireev, A. S.; Kornienko, A.; Org. Lett. 2006, 8, 899.
3. Triggle, D. J.; Mini-Rev. Med. Chem. 2003, 3, 215; Triggle, D.; J. Cell. Moll. Neurobiol. 2003, 23, 293.

4. Boschi, D.; Caron, G.; Visentin, S.; Di Stilo, A.; Rolando, B.; Fruttero, R.; Gasco, A.; Pharm. Res. 2001, 18, 987.

5. Minsane, I.; Klusa, V.; Dambrova, M.; Germane, S.; Duburs, G.; Bisennieks, E.; Roimondini, R.; Ogren, S. O.; Eur. Neuropsychopharmacol. 1998, 8, 329.

6. Suárez, M.; de Armas, M.; Ramírez, O.; Alvarez, A.; Martinez, R.; Molero, D.; Seoane, C.; Liz, R.; Novoa, H.; Blaton, N.; Peeters, O.; Martín, N.; New J. Chem. 2005, 12, 1567.

7. Suárez, M.; de Armas, M.; Ramírez, O.; Álvarez, A.; MartínezÁlvarez, R.; Kayali, N.; Seoane, C.; Martin, N.; Rapid Commun. Mass Spectrom. 2005, 19, 1906.

8. Suárez, M.; Molero, D.; Salfrán, E.; Martín, N.; Verdecia, Y.; Martinez, R.; Ochoa, E.; Alba, L.; Quinteiro, M.; Seoane, C.; Magn. Reson. Chem. 2001, 39, 105.

9. Suárez, M.; Martín, N.; Martínez, R.; Verdecia, Y.; Molero, D.; Alba,L.; Seoane, C.; Ochoa,E.; Magn. Reson. Chem. 2002,40, 303.

10. Molero, D.; Suárez, M.; Martínez-Álvarez, R.; Verdecia, Y.; Martín, N.; Seoane, C.; Ochoa, E.; Magn. Reson. Chem. 2004, 42, 704 .

11. Salfrán, E.; Suárez, M.; Molero, D.; Martínez-Álvarez, R.; Verdecia, Y.; Ochoa, E.; Álvarez, A.; Seoane, C.; Herrera, A.; Martín, N.; Magn. Reson. Chem. 2006, 44, 637.

12. Berger, S.; Braun, S.; Kalinowski, H. O.; NMR Spectroscopy of the Non-Metallic Elements, John Wiley \& Sons: Chichester, 1997 ( ISBN 047196763 7).

Submitted: March 26, 2010 Published online: July 27, 2010 